\title{
Sobre la administración \\ Comentarios a un texto de Bunge
}

\author{
Juan Manuel Silva Camarena*
}

\section{Resumen}

Con el fin de pensar rigurosamente la cuestión relacionada con la posible cientificidad de la administración nos propusimos elaborar este ensayo, por medio de una serie de comentarios, como una lectura crítica de la ponencia del filósofo de la ciencia argentino Mario Bunge titulada "Status epistemológico de la administración". El escrito de Bunge nos ofrece la oportunidad de examinar con cierto detalle la tesis que desde el principio señalamos como inadmisible: la propuesta de Bunge de que las cuestiones de la administración puedan plantearse y resolverse de manera científica sin que la administración misma sea una ciencia, aun cuando coincidamos plenamente en la idea de que la ciencia es fundamentalmente una versión desinteresada de la realidad.

Palabras clave: administración, ciencia, científico, técnica, teoría y práctica.

$E^{\prime}$ 1 presente trabajo tiene el propósito de contribuir a la aclaración de algunas cuestiones relacionadas con la naturaleza de la administración mediante la presentación de una serie de comentarios al escrito de Mario Bunge titulado "Status epistemológico de la administración"1 . Con el fin de que el lector que la necesite posea una idea general de lo que está en discusión, viene a cuento señalar

\footnotetext{
* Coordinador del Programa de Formación de Investigadores de la División de Investigación de la Facultad de Contaduría y Administración, UNAM. Correo electrónico: philjm01@hotmail.com

${ }^{1}$ Mario Bunge, Licenciado en Ciencias Físicomatemáticas y Doctor en Física, ha dedicado mucho trabajo a enseñar y escribir sobre filosofía de la ciencia. En 1980 presentó una ponencia en las Primeras Jornadas Nacionales de Administración en Buenos Aires con el título de "Status epistemológico de la administración”. Eduardo R. Scarano, profesor de epistemología y metodología de las ciencias sociales de la Universidad de Buenos Aires, incluyó el trabajo de Bunge en una compilación titulada Metodología de las ciencias sociales. Lógica, lenguaje y racionalidad, publicado por Ediciones Macchi, de Buenos Aires, en 1999. La ponencia de Bunge está organizada en cinco apartados: I) el problema, II) ciencia y técnica, III) objeto, medios y meta de la administración, IV) conclusiones y v) referencias bibliográficas (del mismo autor: 1) La investigación científica, Ariel, Barcelona, 1969, Epistemología, Ariel, Barcelona, 1980, y Ciencia y desarrollo, Siglo Veinte, Buenos Aires, 1980. 2) A World of Systems, D. Reidle Publ. Co., Dordrecht-Boston, 1979. 3) A World of Systems, ed. cit. 4) General Systems and Holism, General System, XII, 1977).
} 
desde el comienzo que no consideramos que sea admisible la propuesta de Bunge de que las cuestiones de la administración puedan plantearse y resolverse de manera científica sin que la administración misma sea una ciencia. Nosotros consideramos que sólo bajo la condición de que la administración sea una ciencia pueden abordarse y resolverse sus problemas científicamente. Nuestras observaciones han sido enlistadas con números arábigos y aparecen enseguida del pasaje a que se refieren, mismo que está presidido por una idea orientadora que en cada caso lleva un número romano. Cabe aclarar que el texto del profesor Bunge ha sido presentado y examinado en su totalidad, y que sus diversas partes aparecen en nuestra trascripción en el mismo orden consecutivo de la versión original.

\section{Bunge comienza a averiguar si la administración es una ciencia, a partir de la consideración de dos maneras distintas de referirse a ella}

En diversos paises latinos funcionan facultades de ciencias de la administración. En cambio, los anglosajones prefieren las denominaciones más modestas Faculty of Management o School of Business and Administration, comúnmente conocida por BA School. ¿Se tratará de un ejemplo del amor latino por la hipérbole, o de un error, o bien será que, en efecto, los estudios de administración constituyen una ciencia? Veamos [p. 351]².

1. El punto de partida de la averiguación de Bunge, el que se refiere al supuesto amor latino por la hipérbole, es una exageración o es trivial. También podría considerarse que en México, en la Universidad Nacional Autónoma de México, a la administración se le enseña en la Facultad de Contaduría y Administración, y que en el Canadá francófono se denomina École de Gestion, mientras que en Alemania... El pensamiento filosófico no se ve obligado a buscar el estatuto epistemológico de la administración por el hecho de que unos dicen que es ciencia y otros dicen lo contrario (como si fuera un enderezador iluminado de las opiniones de los hombres). Esta cuestión se la plantea la investigación filosófica como una tarea de su propio trabajo (como la química se compromete, por su objeto de estudio, a distinguir la naturaleza química de los elementos). Es normal que la investigación filosófica (en el quehacer de la teoría del conocimiento o la epistemología) incluya entre sus cuestiones fundamentales el problema de la diferencia

\footnotetext{
2 Todos los pasajes del escrito de Bunge (que conforman la totalidad del texto) se han tomado literalmente de la edición mencionada de E. R. Sacarano.
} 
Sobre la administración. Comentarios a un texto de Bunge

entre el conocimiento ordinario o precientífico y el conocimiento científico, y así se ve obligada a dar razón del estatuto epistemológico de esta forma de praxis humana que llamamos administración.

2. Si se tratara de una diversidad de denominaciones y horizontes culturales se nos invitaría más bien a plantear una pregunta como ésta: ¿es posible encontrar diferencias radicales en la concepción de lo que es la ciencia en las tradiciones académicas latinoamericana, anglosajona y europea? En el caso de una respuesta afirmativa se abriría sin duda una brecha inquietante de investigación.

\section{Bunge asegura que algunos filósofos saben que la administración su- frió una revolución después de la Segunda Guerra Mundial}

Todos, excepto quizá los filósofos de corte tradicional, estamos enterados de que los estudios de administración han sufrido (o gozado) una revolución desde fines de la Segunda Guerra Mundial, gracias a la investigación operativa, la modelización matemática, un contacto más estrecho con la psicología y las ciencias sociales, y la informática [p. 351].

No es posible establecer con rigor una distinción entre filósofos de corte tradicional y otros de corte no tradicional — ¿modernos? En todo caso ésta es una caracterización sin consecuencias filosóficas que sólo comunica, sin ningún contenido significativo preciso, un modo personal y subjetivo de referirse a (y de concebir) el trabajo de la filosofía.

1. La averiguación teórica sólo puede partir de lo que de hecho es el conocimiento en la teoría y la práctica administrativas. No se puede tomar en serio el supuesto saber que compartirían sólo algunas personas que afirmaran estar enteradas de un asunto. La administración ha sufrido una revolución en su evolución o no la ha sufrido. Y si fuera una cuestión de hecho ya no importaría que lo supieran unos filósofos y otros lo ignoraran.

El sentido preciso de lo que significa una revolución en el conocimiento científico o filosófico (y por tanto, la posibilidad de utilizar esta noción para caracterizar un fenómeno de conocimiento) sólo puede quedar suficientemente justificado en una adecuada fundamentación teórica. De otro modo, sólo se justificaría la afirmación - y a medias - en un texto de divulgación de la ciencia que tuviera la misión de exaltar el fenómeno, hiperbólicamente, en las mentes juveniles. 
2. El concepto de revolución científica, si no se queda en una mera metáfora, es algo mucho más complejo de lo que parece. Una revolución en la ciencia no se produce solamente porque en un determinado campo de conocimiento se adopta algo nuevo (una tesis, una hipótesis, un estilo de trabajo, técnicas, procedimientos o conocimientos de otras disciplinas científicas, etcétera), sino cuando en dicho campo tiene lugar un cambio radical, fundamental, que trastorna una respetada y respetable tradición científica, que impide que en lo sucesivo sea posible conseguir conocimientos válidos como si nada hubiera pasado. Un ejemplo de revolución científica lo representa la aparición del psicoanálisis y su concepto de inconsciente, que obligó a reconsiderar seriamente si la vida psicológica podía concebirse como el ámbito exclusivo de la conciencia.

\section{Según Bunge la administración pasó ya del trabajo meramente empí- rico a la etapa científica, y por eso puede encarar y resolver sus cuestiones de manera científica}

Esta revolución ha marcado la transición de la empiria a la etapa científica en este campo de estudios y de actividades. Basta hojear un texto moderno de contabilidad, planeación u organización empresarial, o un número de Management Science, para advertirlo. Admitiremos, en suma, que los estudios administrativos han alcanzado un nivel cientifico [p. 351].

1. No se puede admitir de un modo filosóficamente fundado que los estudios administrativos han alcanzado un nivel científico mediante la hojeada de ningún texto, sea antiguo o moderno.

2. Tampoco se puede proporcionar un testimonio serio del nivel científico de los estudios administrativos echando una mirada a una revista, aunque esté escrita en inglés y esté dedicada a la divulgación de trabajos serios sobre la administración (cuyo nivel de rigor científico, desde luego, es necesario mostrar en cada caso).

3. Es arbitraria la decisión de admitir un supuesto nivel científico de los estudios administrativos sin investigación alguna y sin recurrir a la fuerza mostrativa o demostrativa de la argumentación. En este caso esa admisión no puede expresar sino una opinión personal carente de fundamento.

4. Sin razones suficientemente fundadas, es imposible admitir o rechazar nada en el trabajo científico de la filosofía, que por cierto siempre es algo más que un juicio personal, superficial e inconsistente. 
Sobre la administración. Comentarios a un texto de Bunge

\section{Para Bunge hay una diferencia entre el adjetivo científico y el sustan- tivo ciencia}

Sin embargo, el problema propuesto no se resuelve con sólo reconocer que las cuestiones administrativas pueden encararse y resolverse de manera cientifica. Subsiste la diferencia entre el adjetivo científico y el sustantivo ciencia [p. 351].

1. No se trata de una diferencia real que haya que reconocer o tener en cuenta, sino de lo que hay que mostrar. Lo que tiene que probarse precisamente es que las cuestiones administrativas pueden encararse y resolverse de manera científica. Dicho de otro modo, no puede solucionarse el problema de si la administración es una ciencia o no lo es si se supone, sin mostrarlo, que las cuestiones administrativas pueden encararse y resolverse de manera cientifica.

2. No puede reconocerse lo que no ha sido previamente demostrado, a menos que se invoque a la autoridad de quien sostiene tal reconocimiento.

3. Sin duda es una afirmación extraña la que sostiene que algo participa de la naturaleza de algo sin ser o llegar a ser de la misma naturaleza. ¿En verdad se puede afirmar con sentido que hay algo que es científico y sin embargo no es ciencia y sí pertenece a la ciencia?

4. Todo parece indicar que el autor del texto se ve obligado a establecer arbitrariamente una distinción entre un adjetivo y un sustantivo para llegar a la conclusión que le parece ingenioso sostener: "hay algo que es científico, pero no es ciencia". Como si lo religioso fuera sólo una forma de religiosidad que nunca pudiera considerarse religión.

5. Se trata del establecimiento artificial de algo que no sería enteramente ajeno a la ciencia, pero que situándose en una especie de punto medio, tampoco llegaría a ser la ciencia misma. Este procedimiento parece saltar fácilmente el obstáculo de la pregunta por la cientificidad de la administración, pero el recurso va a parar a un híbrido imposible, sin realidad alguna.

6. Si la cientificidad de la ciencia se desdobla artificiosamente en dos aspectos, en la ciencia y, como algo aparte, su método, se crea la ilusión de que se trata de dos cosas distintas, y por tanto, separables. Así aparece la idea de que el método científico no es exactamente equivalente a la ciencia, y entonces (paradójicamente) 
puede servir para hacer ciencia y para hacer algo que parece ciencia sin serlo. Sólo mediante este procedimiento puede concebirse un único método cuya finalidad pudiera indistintamente servir para la técnica (conocimiento interesado) y la ciencia (conocimiento desinteresado), dando lugar a lo imposible: lo científico que no es ciencia.

El problema de saber si la administración es ciencia o no lo es no puede resolverse si en la argumentación se introduce subrepticiamente lo que es el objeto de la búsqueda. Esta argumentación sofística —o argumento aparente — se llama petición de principio (petitio principii): cuando se usa para la prueba precisamente lo que debe ser probado. No puede reconocerse lo que es materia de indagación ("que las cuestiones administrativas pueden encararse y resolverse de manera científica").

\section{Bunge quiere determinar si la administración es una ciencia o una técnica}

La fabricación de artefactos cerámicos, de vidrio o de acero es hoy en día un proceso controlado por técnicas que poseen fundamento cientifico; o sea, se funda sobre resultados de investigaciones físicas y químicas, además de emplear conocimientos obtenidos en investigaciones y ensayos técnicos. Pero las técnicas de producción de artefactos cerámicas, de vidrio y de acero no son ciencias, son ramas de la ingeniería. Se trata de averiguar si los estudios de administración, aun los más rigurosamente cientificos, constituyen una ciencia comparable con la química o la sociología, o más bien una técnica comparable con la ingeniería nuclear, la agronomía, la medicina, el derecho o las finanzas [pp. 351-352].

1. Ésta es la pieza falsa: creer que la técnica puede tener un fundamento científico. Esta creencia puede explicarse. Si se considera erróneamente que la ciencia es matemáticas, entonces se llega a pensar que la técnica es científica si se utiliza en su diseño o su realización algún cálculo matemático.

2. La actividad cognoscitiva es ciencia si y sólo si reúne las características esenciales del saber científico, no cuando un conocimiento es algo comparable al saber de la ciencia.

3. Quizá sea conveniente pensar que la alternativa correcta para determinar si la administración es una ciencia o no lo es no consiste en decidir si es ciencia o técnica, sino en averiguar si ella es conocimiento científico o conocimiento no 
Sobre la administración. Comentarios a un texto de Bunge

científico (independientemente de que éste incluya o no técnicas para resolver problemas prácticos). No se trataría de saber si la administración es ciencia o técnica, sino de averiguar si es ciencia o no lo es.

4. No existen técnicas con fundamento científico o sin él. El fundamento científíco sólo es fundamento para el conocimiento científico. De la técnica no se puede predicar la verdad o la falsedad, sino su eficiencia o ineficiencia para lograr un determinado resultado. Ningún tipo de técnica necesita fundamento teórico: funciona o no funciona, independientemente de consideraciones teóricas. Se trata de dos formas de acción humana distintas.

La técnica, como respuesta ante la necesidad, existe en las comunidades humanas desde tiempos remotos, en las que nunca hubo ciencia. Donde nace la cienciaen Grecia-, la técnica existe muchísimo tiempo antes del surgimiento del saber científico. Los hombres saben hacer muchas cosas, con técnicas primitivas o refinadas, antes de plantear la pregunta por lo que esencialmente son todas las cosas (si aire, si agua, si fuego...). Si se confunde la técnica con la ciencia carece de sentido hablar del nacimiento de la ciencia. Ésta sería lo mismo que aquélla, con un poco más de complejidad y sofisticación.

\section{Bunge cree que el problema del estatuto filosófico de la administra- ción tiene un interés filosófico. Apartado 1}

Este problema del status epistemológico de la administración es de interés filosófico, ya que le da al filósofo la oportunidad de afilar sus ideas acerca de la ciencia y la técnica, así como de aplicarlas a un campo en pleno desarrollo, con la esperanza de ser de alguna utilidad a sus cultores. Pero ¿tiene interés práctico, aparte de amenazar a las administraciones universitarias con un costoso cambio de rótulos? Aparentemente no: parecería ser uno de esos problemas académicos cuya solución tiene "solamente" el mérito de aclarar algunas ideas $y$, por lo tanto, no será estimado por quienes teniendo ideas confusas o contadas, no las valoran sino como herramientas para la acción [p. 352].

1. El interés filosófico del estatuto epistemológico de la administración no depende de las oportunidades que pueda ofrecer o negar a los filósofos para verificar si sus ideas acerca de la ciencia y la técnica son correctas o erróneas. Considerar que es bueno que exista la administración como objeto de examen filosófico para confirmar o corregir nuestros conocimientos acerca de la naturaleza de la ciencia es 
algo no filosófico. No es lo mismo que algo tenga un interés filosófico que algo sea de interés para un filósofo.

2. La investigación filosófica no sale particularmente beneficiada cuando averigua la naturaleza epistemológica de esa particular forma de praxis humana que es la administración. La filosofía nunca trabaja con la esperanza de ser útil para tareas ajenas a su propio quehacer. El éxito de la investigación filosófica depende del rigor y la exactitud con la que ella puede determinar el estatuto epistemológico de esta forma de acción humana, independientemente de alguna eventual utilidad práctica que pudiera derivarse de dicha determinación. El mérito de aclarar ideas principales no es para la filosofía algo que pudiera palidecer frente a un atractivo conjunto de ganancias prácticas.

3. Los científicos no están interesados en ser útiles para un gremio o para otro, para una institución o para otra: saben bien que son útiles para la comunidad en su búsqueda desinteresada de la verdad.

\section{Bunge considera que el problema del estatuto filosófico de la adminis- tración tiene un interés práctico}

Si se mira más de cerca, se advierte que el problema tiene también algún interés práctico, al menos tanto como saber si la medicina es una ciencia o una técnica. En efecto, si la administración se considera como una ciencia (social), entonces su objetivo central debe ser buscar las leyes y normas que satisface o debiera satisfacer la actividad administrativa, desde el contador hasta el gerente de producción y el encargado de relaciones públicas [p. 352].

1. En las líneas del autor no aparece aclarado en qué consiste el interés práctico de la averiguación del estatuto epistemológico de la administración (no basta con decir que es mayor o menor que otros intereses semejantes).

2. Aunque los problemas prácticos pudieran tener o suscitar un interés teórico, los intereses teóricos no tienen ningún interés práctico. Se trata de intenciones de distinta naturaleza.

3. La seguridad que suelen alcanzar los conocimientos prácticos, configurando o no diversas técnicas, es suficiente para la confianza que se deposita en ellos. 
Sobre la administración. Comentarios a un texto de Bunge

4. El principal interés práctico de la medicina no consiste en saber si es una ciencia o una técnica (éste es un interés teórico), sino en contar con su ayuda eficiente para la curación de enfermedades y la salvación de vidas humanas (o animales, en el caso de la veterinaria).

5. El interés práctico por la medicina y la administración se centra directamente en la cuestión de su eficiencia. La verdad científica que una y otra puedan ostentar no remedia el fracaso del funcionamiento de una organización o la muerte de un paciente.

6. La investigación científica consiste en determinar las razones por las cuales una cosa es como es o explicar por qué sucede lo que sucede. La legalidad o racionalidad que rige el conjunto de acontecimientos y las cosas aparece precisamente en las razones conseguidas por la ciencia.

7. El conocimiento de la praxis administrativa tendrá que clasificarse exclusivamente bajo el rubro de los conocimientos prácticos si no se logra establecer de algún modo claramente identificable el conjunto de los fenómenos propiamente administrativos precisando rigurosamente sus características.

8. La psicología, por ejemplo, existe porque existen fenómenos psicológicos que no pueden ser explicados por el saber científico de otros campos del conocimiento. ¿Los fenómenos de la administración reclaman igualmente un campo propio de conocimientos? Si la legalidad de la actividad administrativa fuera revelada en las razones proporcionadas por la investigación científica en otros campos del saber, la administración se quedaría sin una tarea teórica propia, y por tanto, sin la posibilidad de cumplirse como investigación científica. Si los hechos y las acciones que conforman la praxis administrativa pueden explicarse en su totalidad con los recursos de conocimiento de las disciplinas científicas conocidas, fracasa el proyecto de una ciencia de la administración.

Para que la administración se constituya en una actividad científica necesita contar con un campo propio de conocimientos, aunque para la realización de sus tareas reciba — como las demás ciencias — el auxilio de otros conocimientos teóricos y prácticos.

9. Si los fenómenos de la praxis administrativa no poseen una naturaleza propia, de manera que pueda hablarse con sentido de la realidad administrativa, no hay la 
posibilidad de construir un campo particular de conocimientos. Es necesario que los fenómenos de la administración, como totalidades, sean susceptibles de explicación científica. En sí mismos, como fenómenos aislados, un taco o la producción de bolillos no requieren de explicación científica alguna. Los casos particulares sólo pueden aspirar a una explicación objetiva desde la perspectiva general o universal del enfoque científico.

10. Es necesario distinguir la naturaleza de los problemas. La solución de problemas científicos representa siempre la apertura de una vía de comprensión. En cambio, al resolver un problema práctico se inaugura invariablemente una vía de acción posible.

11. La utilidad práctica, económica o política es algo enteramente ajeno a la tarea de la investigación de la verdad.

En cambio, si la administración es una técnica, dejará esa investigación básica a las ciencias sociales básicas o puras, para ocuparse en cambio de diseñar modelos de organización óptima (en algún aspecto) sobre la base de conocimientos adquiridos en las ciencias básicas, así como de nuevos conocimientos adquiridos en el curso de la investigación y la experiencia administrativas [p. 352].

1. La razón calculadora que funciona en la consecución de resultados prácticos marcha más rápido y ligera e independientemente de la razón científica, la que intenta explicar lo que son las cosas en sí mismas. La administración puede dedicarse exclusivamente al diseño de modelos de organización eficiente (sin la ayuda de conocimientos adquiridos por las ciencias), como ha venido sucediendo en el curso de la historia de la humanidad. Pero también podría aspirar a dar razón teórica, estrictamente científico, de los fenómenos administrativos de las organizaciones.

Existe en nuestros días un malentendido, por un lado, formado por el prejuicio de que se puede y se debe justificadamente despreciar los conocimientos prácticos que no alcanzan el nivel de la ciencia; y por otro lado, promovido por la creencia pertinazmente sostenida - - en verdad nunca cuestionada - de que la técnica (quién sabe desde cuando) recibe el apoyo del conocimiento científico, como privilegio exclusivo, que le permite alcanzar su envidiable eficiencia. 
Sobre la administración. Comentarios a un texto de Bunge

\section{Bunge aborda el tema de la enseñanza de la administración}

A esta división del trabajo le corresponde una divergencia de los planes de enseñanza. Si la administración es una ciencia, entonces debería enseñarse como una mera especialización de las ciencias sociales. En cambio, si es una técnica, se justifica que se enseñe en una facultad especial, donde los alumnos sean expuestos desde el comienzo a cuestiones de administración. En resumen, nuestro problema tiene interés tanto teórico como práctico. Intentemos, pues, resolverlo [p. 352].

1. La enseñanza de la administración depende, en efecto, de lo que se entiende por administración. La enseñanza y el aprendizaje de la administración representan un problema complejo que exige claridad en el discernimiento de lo que en la administración hay de conocimiento científico, de conocimiento práctico, de habilidad técnica, arte o sabiduría directiva (rectoría, dirección y liderazgo de grupos humanos). El saber científico de la administración no se puede enseñar de la misma manera que se enseñan las habilidades prácticas, técnicas, del oficio o profesión de administrador.

Una idea sencilla al respecto organizaría la enseñanza de la ciencia administrativa en la universidad, y la formación del oficio de administrador en alguna escuela especial dedicada al manejo de las empresas. Pero como la administración se ejerce también en organizaciones no empresariales (como las instituciones universitarias y las instituciones de beneficencia), el oficio de administrador se acerca más al conocimiento científico de la administración que a la formación de empresarios (propia de escuelas de negocios), y de este modo se acomoda como sucede en nuestro país - en una facultad universitaria. La universidad contemporánea, de hecho, aloja por igual al cultivo de la ciencia y la investigación científica que a la formación profesional. En ambos casos, empero, se enseñan y se aprenden habilidades técnicas. No sale sobrando la aclaración de que la habilidad técnica, aunque implica conocimientos prácticos, no es una forma de conocimiento, sino un modo de manejar y controlar aspectos de la realidad. Los conocimientos prácticos, que son los que consisten en saber qué hacer para conseguir un determinado efecto, no constituyen por sí mismos una técnica sino hasta que quedan sometidos a una organización u ordenamiento sistemático en función de su efectividad, probada empíricamente, para producir resultados esperados. El objetivo perseguido, en todo caso, obliga a adoptar determinadas estrategias de enseñanza, aprendizaje y aplicación. 
2. Es vaga la idea (y puede revelar cierto practicismo en su concepción) de que los alumnos de administración debieran ser expuestos desde el principio a los asuntos de la administración. ¿Acaso los estudiantes de física o los de filosofía no deben ser expuestos desde un comienzo a las respectivas cuestiones de la física y la filosofía?

3. ¿Qué podría entenderse por una facultad especial, de un modo académicamente justificado dentro de la administración de las universidades? ¿Se sugiere acaso que los conocimientos prácticos debieran enseñarse en escuelas y centros de adiestramiento o entrenamiento y no necesariamente en recintos universitarios?

\section{Para Bunge no son lo mismo la ciencia y la técnica}

La técnica moderna ha alcanzado un nivel tan elevado que a veces es difícil diferenciarla de la ciencia. Con todo, las diferencias existen e importa conocerlas si se ha de impulsar (u obstaculizar) sus desarrollos respectivos. Empecemos, pues, por esbozar sus diferencias por debajo de sus similitudes reales y aparentes (nota 1), [p. 352].

1. La diferencia entre ciencia y técnica tiene un carácter teórico y depende de estas preguntas: ¿cuál es la naturaleza del conocimiento científico?, ¿cuál es la naturaleza del conocimiento técnico? La idea de que una u otra hayan alcanzado o no un determinado nivel depende del criterio que se aplique para la determinación del mismo.

2. Es difícil encontrar un interés práctico a la diferencia entre ciencia y técnica. En todo caso, si la diferencia pudiera tener un interés práctico, no puede decirse que tenga una consecuencia práctica. Para decirlo hipotéticamente: no sucedería nada si la ciencia fuera algo distinto de la técnica (lo cual es cierto).

3. Puede plantearse una pregunta inquietante: ¿Quién tiene autoridad en una determinada comunidad para impulsar o detener el desarrollo de la técnica o de la ciencia?

4. Cabe hacer notar que la diferencia entre la ciencia y la técnica no puede explicarse prácticamente, aunque se llegue a sostener que tiene aplicaciones prácticas. La diferencia exige una explicación teórica. En cuanto tal, esa diferencia tiene un interés teórico en sí misma, pues permite dar razón de la naturaleza del 
Sobre la administración. Comentarios a un texto de Bunge

conocimiento. El interés por las formas de conocimiento se justifica por sí mismo, independientemente de que pueda utilizarse o no en la práctica.

5. ¿Cuál es el sentido de la elevación de la que habla el autor? ¿Se trata de una jerarquía de conocimientos cuya cima corresponde al saber científico? Digámoslo así: ¿si se sigue elevando la técnica un día podría volverse ciencia? Esto no tiene sentido.

6. Es muy probable que la carencia de una idea clara acerca de lo que es el conocimiento práctico y el conocimiento científico oscurezca el concepto mismo de técnica, dando lugar a confusiones inevitables.

7. Entre la ciencia y el conocimiento práctico hay una diferencia vocacional radical, que se expresa claramente en sus respectivas intenciones, saber qué es la cosa o saber qué se puede hacer con ella. Esto no impide la existencia de una similitud innegable: ambas son formas de conocimiento que el hombre pone en acción en términos de averiguación. La técnica, como conocimiento ya logrado y probado que el ser humano aplica en un orden o curso regular para obtener determinados resultados previamente establecidos, no es conocimiento teórico sino práctico.

\section{Bunge propone una distinción entre ciencia básica y ciencia aplicada}

Ante todo conviene distinguir las ciencias básicas (o puras) de las aplicadas. La diferencia no es de método, sino de meta y, por lo tanto, de producto. La investigación básica se interesa por problemas cognoscitivos de cualquier tipo; la aplicada por problemas cuya solución tiene alguna posibilidad de utilización práctica, sea económica o política. El científico básico se esfuerza por encontrar las leyes básicas de la realidad; el aplicado, por aplicarlas. Ambos utilizan el método científico; ambos hacen uso de cuantas ciencias sean necesarias y ambos proveen conocimiento nuevo. Pero, mientras que el primero se propone solamente entender la realidad, el segundo busca entender una parte de ésta para que alguien pueda transformarla [p. 353].

1. No está suficientemente justificada la distinción entre ciencias básicas y ciencias aplicadas. Sólo existen, por un lado, ciencias y, por el otro, conocimientos prácticos. Dentro de estos últimos hay procedimientos técnicos para una y mil cosas (en la industria, la producción de alimentos, el arte, la ciencia, la medicina, etcétera). 
2. Los conocimientos prácticos no adquieren carta de naturalidad por medio de una honorabilidad científica agregada. Su naturaleza, por sí misma, por sus fines y medios, está suficientemente justificada en términos cognoscitivos, y no siempre en términos de censura moral.

3. En sentido estricto, ningún científico se dedica "a aplicar" conocimientos científicos, cuando toma en cuenta otros conocimientos, teóricos y prácticos, lo hace solamente para conseguir nuevos conocimientos teóricos. El hombre práctico es suficientemente práctico como para no confiar en una aplicación de conocimientos científicos, abstractos e hipotéticos, cuando le importa de verdad el resultado, no la verdad de los enunciados (para la satisfacción de una necesidad cotidiana o un asunto de vida o muerte).

4. El método científico, como lo sugiere su mismo nombre, sólo se puede usar para una sola cosa: para hacer ciencia. Pero el método científico no es, a su vez, una técnica (conocimiento ya obtenido y probado) para la consecución de conocimientos.

El método científico no es una técnica, sino un modo de investigar la realidad - con objetividad, racionalidad y sistema - propio del trabajo cognoscitivo de las ciencias naturales y sociales. En este sentido nunca es la mera utilización de un camino ya recorrido. La ciencia es exploración original del camino (sea cual fuere su objetivo).

5. La exploración es vigilada. En el método científico, la objetividad se logra con la vigilancia ética, lógica y epistemológica.

6. Es un desatino pensar que el llamado método científico sirve para atender tareas de conocimiento no científico, y carece de fundamento la idea de una ciencia que se cultivaría deliberadamente como ciencia aplicada, y que serviría para la solución de problemas prácticos, creando la ilusión de que recibirían de este modo soluciones científicas.

7. Efectivamente, el científico (a secas, como se dice) se esfuerza por resolver problemas teóricos (Bunge dice "por encontrar las leyes básicas de la realidad"); el práctico se encarga de solucionar problemas prácticos mediante conocimientos prácticos, viejos o nuevos. En una división del trabajo que se antoja natural, el práctico nunca se pone a resolver problemas teóricos, como tampoco el científico se dedica a solucionar asuntos prácticos. 
Sobre la administración. Comentarios a un texto de Bunge

8. No existe la profesión del que supuestamente se dedicaría a entender la realidad o una parte de ella "para que alguien pueda transformarla". ¿Quiénes son los que trabajarían arduamente en la investigación científica esperando tanto la verdad en sus descubrimientos como la aparición del práctico que iría a aplicarlos para transformar la realidad?

9. La distinción entre entender o interpretar el mundo y transformarlo (referible a la Onceava tesis sobre Feuerbach de Marx) nació como una denuncia del supuesto origen y uso ideológico del conocimiento, pensando en la transformación política del mundo, y no en una supuesta transformación de la realidad que se lograría "aplicando" conocimientos científicos.

10. El mundo queda humanamente transformado cuando alguien entiende práctica o científicamente las cosas (no cuando se altera lo real supuestamente con conocimientos científicos "aplicados"). En primer lugar, se transforma el científico (pues se libera de la necesidad del saber práctico); y en segundo lugar se transforma la comunidad entera (que de ese modo advierte que puede saberse sin necesidad de utilizar el mundo). El carácter ético de la obtención y el uso del saber es fundamental para la comprensión de lo que es la ciencia, y para distinguirla de otras formas de acción humanas.

La ciencia no tiene dos finalidades, una de entender y otra de utilizar - que el autor llama transformar-, sino una sola: la de saber lo que son las cosas, independientemente de lo que se puede obtener de ellas utilizándolas. La otra finalidad, la práctica, define en cambio otra clase de conocimientos: los de las ideas que sirven para hacer esto o esto otro.

\section{Bunge cree que es posible distinguir la tarea del científico básico, el científico aplicado y el técnico}

Un ejemplo: al par que el sociólogo o el economista básico estudia sociosistemas (sistemas sociales) con el fin de comprender cómo funcionan (bien o mal), el científico social aplicado los estudia con el fin de averiguar qué favorece u obstaculiza su mantenimiento o su desarrollo en algún sentido. Y lo hace con la esperanza (o el temor) de que los resultados de su estudio sean utilizados, por quienes ejercen poder, para modificar dichos sistemas [p. 353]. 
Es una idea sin fundamento la que permite establecer la diferencia entre ciencia básica y ciencia aplicada. Sólo hay ciencia $-\mathrm{y}$ que por tanto no puede llamarse básica respecto a nada- y como ciencia social trabaja para entender individuos y comunidades humanas en su origen, su organización y su desarrollo histórico (que no funcionan bien o mal como las especies animales, los aparatos o las máquinas).

1. Hasta nuestros días, en los que actúa la versión pragmática o utilitaria de la ciencia, las nociones de "lo básico" y "lo aplicado" son categorías que naturalmente no aparecen en la historia de la ciencia.

2. La supuesta diferencia entre ciencia básica y ciencia aplicada remite, en última instancia, a una prejuiciada manera de entender lo teórico y lo práctico, movida por el interés de salvar un sentido práctico de la ciencia, el cual, a su vez, salvaría a la ciencia misma de una supuestamente perniciosa concepción que mediante la idea del desinterés práctico la colocaría, a través de una perorata insufrible, en el "pedestal" del saber por el saber, cuya mera existencia representaría la ofensa de una gran traición al hombre mismo.

3. Las preocupaciones ajenas a la investigación científica, extrañas a la búsqueda de la verdad como las que pueden aparecer en las consideraciones de políticos, empresarios e industriales, ¿tienen que adquirir un espacio dentro del quehacer mismo de la ciencia?

El técnico, en cambio, puede investigar o utilizar los resultados de investigaciones (propias o ajenas), pero en ningún caso se queda en el conocimiento: aspira a poner el saber en acción. El centro de la actividad cognoscitiva técnica es el diseño de dispositivos o planes de acción que permitan crear o controlar cosas concretas. Los objetos de este control pueden ser físicos (centrales eléctricas), químicos (plantas petroquímicas), bioquímicos (bodegas), económicos (supermercados), culturales (museos) o políticos en sentido amplio de la palabra (prisiones) [p. 353].

1. El conocimiento también es una acción. Desde el punto de vista de la fundamentación de las ideas es bastante ingenua la división entre conocimiento y acción, nacida en como una distinción práctica, no teórica, que manifiestamente sólo tiene sentido fuera del ámbito de la ciencia.

2. El técnico no es el hombre cuerdo que por gracia del sentido común se escapa de la abstracción y aspira a poner el saber en acción, rescatándolo de las redes del 
Sobre la administración. Comentarios a un texto de Bunge

conocimiento desinteresado con las que pesca sus nociones la retrógrada metafísica. Aspira a solucionar problemas concretos como cualquiera que necesite resolver un problema práctico para satisfacer una necesidad, una ambición o un deseo.

3. El saber de la técnica es un saber con dignidad cognoscitiva propia, cuyos valores (positivo y negativo) no son la verdad y la falsedad, sino la eficacia y la ineficacia.

4. El ideal positivista de saber para prever, y prever para actuar puede adoptarse y se adopta a menudo como divisa para la vida en alguna concepción del mundo, pero es enteramente inadmisible como legítimo lema de la actividad científica.

5. El técnico es sencillamente el que sabe cómo hacer algo o utilizar algo para que suceda algo determinado. Eso es todo. El que sabe usar una herramienta, el que opera una máquina, el que maneja un aparato o un dispositivo.

6. El que fabrica los dispositivos técnicos creando su uso es lo que siempre se llamó con el adecuado nombre de inventor: un hombre práctico que resuelve problemas prácticos. El hombre de ciencia, en cambio, no inventa aparatos sino teorías, o sea, las crea en su búsqueda de la verdad de lo que es, lo que cambia y lo que sucede. ¿Por qué tendríamos que renunciar a nociones tan sencillamente correctas?

El científico, sea básico o aplicado, se propone averiguar cómo son las cosas. El técnico inventa cosas nuevas o bien la manera de controlar (manejar, administrar, mantener, mejorar o destruir) cosas ya conocidas. Si el técnico obra científicamente, se sirve para ello de conocimientos cientificos, aunque no de los más generales y profundos, sino de los que necesite para lograr su objetivo; estos conocimientos utilizables resultan ser casi siempre especificos (poco generales) y menos profundos que los que busca el cientifico básico. En una palabra, mientras que el científico, sea básico o aplicado, busca la verdad (para todos), el técnico se sirve de ésta para alcanzar la utilidad (para alguien) [p. 353].

1. El científico, en efecto, "se propone averiguar cómo son las cosas". Ya señalamos por qué no cabe la distinción de "básico" y "aplicado". Como no puede haber científico "aplicado", tampoco puede haber un técnico capaz de obrar científicamente porque "se sirve de conocimientos científicos". Los conocimientos cien- 
tíficos se consiguen y sirven para "averiguar cómo son las cosas". Una averiguación de esta naturaleza no le sirve al trabajador de la técnica.

2. Si fuera cierto que el técnico usa conocimientos científicos (según el autor los conocimientos científicos superficiales y particulares son los que utiliza el técnico), ¿no sería mejor que sucediera lo contrario? ¿Por qué no servirían para este cometido los conocimientos profundos y universales de la ciencia? Resulta desafortunada, pues, la imagen de lo profundo y lo superficial para establecer una relación entre el saber científico y el técnico.

3. El razonamiento es correcto: la ciencia busca la verdad; el conocimiento práctico procura la utilidad del resultado. El técnico obra técnicamente; el científico, científicamente. No es conveniente torcerle el cuello a las palabras.

\section{Bunge adopta una vieja cuestión: ¿entender el mundo o transformarlo?}

En resumidas cuentas, en tanto que la ciencia se propone explicar el mundo, la técnica se propone forjar las herramientas necesarias para transformarlo. (La transformación propiamente dicha es obra de la acción, sea económica, política o cultural.) Esta caracterización general de la técnica, unida a cierta clasificación de las cosas de este mundo (nota 2), sugiere clasificar las técnicas como sigue:

- fisiotecnias: las ingenierías clásicas;

- quimiotecnias: química industrial, ingeniería química;

- biotecnias: medicina, agronomía, farmacología, pedagogía, etc.;

- sociotecnias: derecho, finanzas, administración, etc.;

- técnicas generales: informática, cibernética, etc.

Me he pisado; ya he dicho dónde coloco la administración. Trataré a continuación de justificar esa ubicación [pp. 353-354].

La exposición de este pasaje tiene el tono de una apología indecisa de la técnica. Como defensa a medias de la técnica es apologética porque exalta su valor al destinarle el decisivo papel de la transformación del mundo, frente a una tarea de menor categoría que sería la propia de la ciencia - explicar el mundo-, y sin embargo, el aplauso no suena fuertemente porque a la técnica se le exhibe como utilizadora superficial de la verdad (del trabajo de otros, digamos) para el logro, muy a menudo admirable, de las transformaciones útiles. 
Sobre la administración. Comentarios a un texto de Bunge

1. Con ciertas aclaraciones de por medio, se puede estar en acuerdo completo con el autor: la ciencia explica el mundo, la técnica lo transforma. Una es teoría; la otra, acción práctica. El siguiente paso es el que no podemos dar con el autor: el de la unidad de la transformación práctica explicativa. La técnica no explica, porque la técnica no opera científicamente.

La metáfora que utiliza el autor — la del pisarse a uno mismo — podría revelar en cierto modo o indirectamente el atropellamiento que se comete con lo real cuando se le aplican ideas preconcebidas. Ella podría sugerir la arbitrariedad que se comete cuando se trata de imponer artificialmente un esquema preconcebido de las ciencias en lugar de explicar cómo es realmente la naturaleza del saber científico.

Parece que a la técnica se le tendría que convertir en una especie de "ciencia chiquita" — como una ciencia a medias, si se pudiera decir-, para acercarla a la idea artificial de una ciencia práctica.

2. No hay duda de que la técnica es instrumento de transformación de la realidad. Pero es conveniente insistir en que la comprensión de lo real que realiza la ciencia provoca igualmente una transformación del mundo (de lo humano) no a pesar de sino precisamente por el desinterés de su conocimiento.

3. La acción no se reduce a la acción práctica. ¿Si se entiende correctamente que la teoría también es una acción, que se concreta en eso que llamamos ciencia, podrían los prácticos (y sobre todo los practicones) aterrados entrever la pérdida irreparable del monopolio de la acción?

4. Ningún científico pretende entender una parte de la realidad para que otra persona pueda transformarla. Suena casi como aquello de que a veces hay alguien que paga la música y resulta que otro la baila. Esto sólo puede corresponder a una organización artificial del conocimiento y la acción.

5. Parece que a como dé lugar a la ciencia se le quisiera hacer práctica, y al conocimiento práctico se le quisiera adornar con los atributos del conocimiento científico. ¿No hay gato encerrado en todo esto?

\section{Bunge reflexiona sobre la relación entre sistemas sociales y administración}

Todo sociosistema humano se caracteriza por su composición (las personas que forman parte de él), su ambiente (natural y social) y su estructura (el 
conjunto de las relaciones entre sus miembros y entre éstos y objetos ambientales). Es común que una persona pertenezca a varios sociosistemas: a su familia, la empresa u organismo donde trabaja, su club, su partido político, etc. Cada uno de estos sociosistemas desempeña funciones que le son propias; estas funciones o actividades forman parte de la estructura del sistema (nota 3) [p. 354].

1. No hay que perder de vista el hecho de que todo sistema de organización de hombres es una comunidad humana; que las organizaciones de animales son especies biológicas y que los sistemas electrónicos son sistemas de naturaleza física. Las diferencias ontológicas de las distintas formas de ser de los entes determinan el carácter de sus sistemas.

Por distintos que sean dos sociosistemas, por ejemplo, una fábrica y una escuela, o un hospital y un ejército, comparten diversos rasgos y, en primerísimo lugar, éste: todos los sociosistemas, por sencillos que parezcan, tienen administradores, aunque sea de tiempo parcial. Por ejemplo, normalmente los padres son los administradores de la familia; la administración de una escuela está formada por su dirección y, en los países anglosajones, también por sus maestros y representantes de los padres; la administración de una empresa económica, privada, estatal o de carácter cooperativo, está en manos de su directorio y sus ejecutivos y, en muchos casos, también de representantes de su personal [p. 354].

1. Aquí hay una especie de retórica que confunde al razonamiento. Para que las frases "por sencillos que parezcan" y "aunque sea de tiempo parcial" no sean enteramente superfluas, se les puede eliminar. De otro modo se comete un error lógico elemental al mezclar la noción de administrador con la noción del tiempo en que se ejerce, como si éste tuviera que ver con la naturaleza de aquello. Pero la administración es administración, independientemente del tiempo usado por el administrador para administrar (sea cual fuere el tiempo utilizado en la administración). En términos aristotélicos, no se distinguiría el nivel de un accidente y una sustancia. Algo similar sucede con lo que se afirma de los "sociosistemas", que requieren de administraciones, a pesar de su aparente sencillez.

2. La tarea de la administración no cambia de naturaleza cuando cambian los actores que la ejecutan. ¿O se quiere sugerir acaso que se trata de un quehacer que lo puede realizar cualquiera, sin una identificación profesional bien determinada? 
Sobre la administración. Comentarios a un texto de Bunge

Es una ilusión querer prescindir de la administración y, en particular, de la burocracia, y por lo tanto es una tontería menospreciarla. Incluso las sociedades formadas por monos y otros animales superiores tienen líderes o administradores, permanentes o temporarios, encargados de organizar la división del trabajo y de controlar que se cumplan las reglas. Los humanos tenemos la ventaja de poder estudiar la administración de un sociosistema con el fin de averiguar la manera de optimizar su funcionamiento. También tenemos la desventaja de poder ignorar tales estudios y, sin embargo, mantenernos, por la fuerza o por el poder económico, a la cabeza de algunos sociosistemas. (En la naturaleza, los administradores ineficientes sucumben o son reemplazados, y las formas de organización ineficientes terminan por ser eliminadas por selección natural. Nosotros premiamos a los incompetentes conforme al principio de Peter y protegemos a los improductivos según la ley de Parkinson.) [p. 354-355].

1. No se sabe quién y bajo qué argumentos quiere "prescindir" de la administración. Es incorrecta la asociación entre administración y burocracia. Y en el trabajo filosófico en torno al estatuto epistemológico de la administración es irrelevante la suposición de su menosprecio y la idea de la imposibilidad o posibilidad de prescindir de ella.

2. Es inadmisible y desafortunada la idea de una administración animal. Si el trabajo de la administración pudiera ser realizado por los animales, entonces no se ejercería bajo la dirección de los atributos humanos. No existiría, para abreviar, la administración de las decisiones libres.

3. Es por lo menos cuestionable la identificación plena entre administración y ejercicio del control y la vigilancia, y es vaga la asociación entre administración, dirección y ejercicio de poder.

\section{Bunge propone un nombre nuevo: la administratecnia}

Puesto que no es deseable prescindir de la administración, es necesario optimizarla. De esto se ocupa, precisamente, la administratecnia. Y, ya que ésta se propone controlar cosas concretas de cierto tipo (sociosistemas), no es una ciencia, sino una técnica, según la definición propuesta en el § 17,2. $Y$, dado que en nuestro tiempo esta técnica no es empírica, sino que se funda sobre resultados de investigaciones cientificas, se trata de una técnica cien- 
tífica al igual que la ingeniería química o la fitotecnia. De hecho, la administratecnia es mucho más cientifica que el derecho y aun que la economía, disciplinas aún fuertemente influidas por la ideología [p. 355].

1. Determinar si la administración es una práctica humana de la que se puede o no prescindir en la vida humana de las comunidades no es una cuestión ni directa ni indirectamente relacionada con el estatuto epistemológico de la administración.

2. Hay que tomar en cuenta siempre que la administración, como investigación científica, no participa, por principio, de la finalidad controladora de algunas prácticas administrativas.

3. Toda técnica, por complejo que sea el caudal de sus conocimientos, es algo ajeno a la ciencia, porque se trata, entre otras cosas, de la aplicación de un conocimiento práctico ya obtenido, no una pregunta sino una respuesta organizada sistemáticamente, mientras que la ciencia es fundamentalmente interrogación, búsqueda, investigación y permanente revisión crítica o autocrítica. No está de más insistir: la técnica funciona independientemente del saber de la ciencia, y se pone en acción antes y más rápidamente que la investigación científica.

4. Validar la expresión técnica cientifica sería anular el criterio vocacional distintivo del conocimiento práctico y el conocimiento teórico, que el propio autor reconoce en la conclusión, y por esta razón se crearía el híbrido inadmisible de un saber al mismo tiempo desinteresado e interesado.

5. Las técnicas (conocimientos prácticos) que se puedan poner al servicio de las investigaciones de las ciencias naturales y sociales no se desnaturalizan en el núcleo mismo de su estatuto epistemológico, y por tanto, siguen siendo técnicas, sin la pretensión de haber quedado conveniente, privilegiada y oportunamente contagiadas de cientificidad.

6. La ciencia, por el contrario, no se puede poner al servicio de la técnica, porque la necesidad que a ésta pone en juego persigue celosamente su eficacia y le impide, por tanto, depender de resultados teóricos que en su última instancia son provisionales e hipotéticos (la teoría es creación siempre sujeta a revisión crítica y autocrítica), y además porque las más de la veces la urgencia de la aplicación desaconseja esperar con paciencia el paso naturalmente lento de la investigación científica. En el conocimiento práctico no hay tiempo que perder, mientras que 
Sobre la administración. Comentarios a un texto de Bunge

en el teórico no domina ninguna exigencia de reloj o calendario. Dicho de otro modo: las cuestiones prácticas de la vida no pueden imprudentemente ajustarse a la lentitud propia del pensamiento científico.

7. Los hechos revelados o explicados en la investigación científica pueden abrir caminos por los que los conocimientos prácticos pueden circular libremente, con independencia o autonomía. Pero los conocimientos científicos, en tanto que teorías o explicaciones desinteresadas de lo real, son inaplicables en el nivel de los conocimientos prácticos.

Para que fuera útil el neologismo arbitrario de "administratecnia" tendría que ir acompañado de una igualmente arbitraria - y por demás superflua - noción de "administraepisteme".

8. Es algo evidente que la naturaleza desinteresada del saber científico podría entrar en conflicto con los intereses políticos de las ideologías. No obstante, ninguna ciencia vocacionalmente bien establecida es vulnerable ante la fuerza de la ideología. Por otro lado, la falta de rigor en la idea de lo que es la ciencia favorece en las opiniones corrientes la influencia de los intereses ideológicos.

\section{Bunge define la administración: una técnica científica}

En resumen, podemos definir la administratecnia, o el sistema de disciplinas que estudian la administración, como la técnica científica que:

a) estudia las actividades y relaciones administrativas que tienen lugar dentro de y entre los sociosistemas;

b) emplea el método científico así como resultados de investigaciones científicas en psicología y ciencias sociales básicas y aplicadas;

c) se propone optimizar en algún aspecto (por ejemplo, productividad, beneficio social o lucro) el funcionamiento de los sociosistemas [p. 355].

1. Si la administración se concibe como el arte de la justa adecuación entre los medios y los fines de la praxis humana, las técnicas de la administración, como las técnicas que sirven al talento musical o pictórico, serían medios para el ejercicio exitoso de esta forma de arte, y la ciencia de la administración, como cualquier ciencia, produciría el saber teórico necesario para dar razón o explicar los fenómenos administrativos. 
Los especialistas distinguirán diversas ramas de la administratecnia, según que se ocupen de hogares, firmas, cooperativas, organismos estatales, escuelas, sociedades privadas de bien público, organismos internacionales, etc. Semejante división del trabajo es razonable, pero no debe llevar a olvidar que todo sociosistema es un subsistema de algún sistema más grande. (Incluso el sociosistema máximo, o sea, el sistema mundial, es un subsistema del sistema solar.) Por lo tanto, no se podrá entender ni, con mayor razón, controlar eficazmente el funcionamiento de un sociosistema, por simple que parezca, si se ignoran sus interacciones con los demás sistemas. Esto no implica que se imponga adoptar un enfoque globalista (holista) y, por lo tanto, antianalitico y, por ende, anticientífico. Significa tan sólo que el enfoque correcto de los problemas administrativos, como el de cualesquiera otros problemas sociales, debiera ser sistémico (nota 4). Lo que no debería extrañar, ya que todo sociosistema es un objeto muy complejo, con múltiples aspectos y modos de cambio, que ninguna disciplina estrecha puede cubrir por entero [p. 355].

No deben confundirse sistemas de naturaleza humana con sistemas de naturaleza física, como si se tratara de naturalezas homogéneas. La dualidad ontológica fijada por la diferencia de lo humano y lo no humano no puede pasarse por alto en la consideración sistemas. La ciencia tiene que cuidarse permanentemente de las falacias, como la que los griegos conocían como una metábasis eis allo genos (o sea en un salto indebido de un género de asuntos a otro). Lo que se predica de una naturaleza no puede predicarse de otra - aunque se les considere en sus interrelaciones sistemáticas - sin tener en cuenta las diferencias de género. Aunque sea posible reducir la totalidad de lo real a la noción de sistema, no pueden pasarse por alto las diferencias ontológicas de los sistemas. El sistema mundial, por ejemplo, es una noción aplicada al ámbito de lo humano que no tiene nada que ver con el sistema solar, que es un concepto que pertenece al ámbito de lo natural.

1. Lo sistemático y lo analítico ni primaria ni necesariamente son aspectos que pudieran entrar en conflicto en el trabajo científico. Además, no hay disciplinas estrechas o anchas, sino disciplinas científicas cuyo objeto de estudio abarca la totalidad de los fenómenos de una misma naturaleza.

\section{Bunge concluye: la administración es científica, pero no es una ciencia}

Las llamadas ciencias de la administración son cientificas por el modo de estudiar su objeto. Pero no constituyen una ciencia, porque, lejos de proponerse alcanzar 
Sobre la administración. Comentarios a un texto de Bunge

conocimientos desinteresados, persiguen conocer la mejor manera de controlar algo, a saber: los aspectos administrativos de los sociosistemas [p. 356].

1. La idea de control abarca cosas y personas. Se pueden controlar fenómenos naturales y sociales. Pero las organizaciones son distintas a los organismos de la naturaleza. En sentido estricto, las organizaciones son comunidades humanas cuya organización es posible sobre la base de la disponibilidad de decisiones para la acción. La estructura misma de la acción permite el acomodo deliberado de los medios y los fines, acomodo que incluye, por supuesto, el control de cosas y personas. Y la mejor manera del acomodo de medios y fines, como arte administrativo (con habilidades y técnicas), admite por otro lado la búsqueda y la consecución de conocimientos desinteresados, que son los que constituyen una ciencia. La naturaleza de las cosas no tiene que ver con la posibilidad o imposibilidad vocacional de su conocimiento desinteresado.

2. Ahora bien, la idea de control de cosas y personas en las organizaciones no es fundamental, sino derivada, no es primaria sino secundaria. Lo fundamental es la adecuación mencionada de medios y fines. El control de cosas y personas para el ejercicio del poder político o económico, por ejemplo, no es exactamente equivalente a la idea central de la adecuación de medios y fines en la organización de las organizaciones (valga la redundancia)

3. La idea central es correcta: la ciencia es un modo de saber que se propone alcanzar conocimientos desinteresados. Este modo, hasta ahora, dentro de la argumentación del autor, no queda imposibilitado. El ejercicio práctico de la administración es otra cosa (y es el que se ha desarrollado predominantemente en la historia de la humanidad, desde la construcción de las llamadas maravillas de la antigüedad hasta la organización administrativa de los vuelos espaciales más asombrosos), e implica, ciertamente, otro modo de conocimiento, que incluye, por cierto, el control de cosas y personas.

4. El desinterés, en efecto, se concibe, desde el Fedón de Platón, como un componente vital de carácter ético que convierte a la averiguación de las razones por las que sucede lo que sucede en investigación científica. Debido a este dispositivo vocacional de búsqueda de la verdad sin segundas intenciones es imposible la confusión posible entre ciencia y técnica.

Que la administración sea una técnica no impide que los administratécnicos formulen modelos matemáticos y diseñen experimentos para poner a prueba 
la verdad de dichos modelos y la eficacia de los controles involucrados. Pero dichos modelos serán específicos o parciales antes que generales. Por ejemplo, se tratará de modelos de la administración de una empresa metalúrgica mediana, o de una línea aérea, antes que de teorías generales acerca de sociosistemas de un género dado. Y se tratará de experimentos cuya finalidad primordial será descubrir fuentes de ineficiencia o mecanismos de optimización de sociosistemas de un tipo bien particular. En suma, la administración es cientifica sin constituir una ciencia. Acaso por este motivo ofrece oportunidades y plantea desafios a personas de origenes e inclinaciones muy diversos, desde el matemático aplicado hasta el conductor de hombres [p. 356].

1. El uso de modelos matemáticos y de cualquier tipo de experimentación son sólo recursos para la prueba decisiva de los dispositivos y las acciones técnicas. Su trabajo cognoscitivo no se logra razonando teóricamente y recurriendo a la experimentación (como en la ciencia), sino razonando calculadoramente y probando.

2. Si los conocimientos técnicos tuvieran que ser obtenidos científicamente (o sea desinteresadamente) no habría técnica. El conocimiento de la técnica es posible porque no tiene que cumplir con ese requisito esencial del saber científico.

3. ¿No es acaso un contrasentido el que conocimientos técnicos, conseguidos interesadamente (como una respuesta ante la necesidad humana), pudieran ser a la vez conocimientos científicos, sin el ingrediente vocacional del desinterés? ¿No es algo sin sentido el pensar que a conocimientos científicos, obtenidos desinteresadamente, se les pudiera sustraer la sustancia vocacional que los generó y pudieran de este modo tornarse en conocimientos prácticos?

4. Efectivamente, el dispositivo vocacional del desinterés es el criterio para determinar la cientificidad o la carencia de ella (el autor dice: no constituyen una ciencia, porque, lejos de proponerse alcanzar conocimientos desinteresados, persiguen...), pero entonces resulta incoherente sostener al mismo tiempo que unos conocimientos fueron obtenidos científicamente y sin embargo no pudieron alcanzar el rasgo de ciencia. No lo pueden alcanzar porque no se lo propusieron desde el principio.

5. El caso particular (esta empresa, y no todas las demás, similares o diferentes), causa en la administración las mismas dificultades que provoca en las ciencias 
Sobre la administración. Comentarios a un texto de Bunge

sociales en general, en las que el ser en cuestión, individual o colectivamente, siempre es un caso único.

6. Si se piensa en otra actividad humana quizá puedan verse más claramente las cosas que nos ocupan. Podría sostenerse que la psicología consigue sus conocimientos científicamente, porque se propone alcanzar conocimientos desinteresados acerca de los fenómenos psicológicos, y señalar que al mismo tiempo no puede ella constituir una ciencia porque se propone controlar (para bien o para mal) el curso de los fenómenos psicopatológicos, en la práctica psicoterapéutica. ¿El autor diría que la psicología no es una ciencia, sino una "psicotecnia"? Tendría que decir que hay ciencia psicológica y psicoterapia.

7. Para poder opinar de una manera similar en el caso de la administración nos hace falta una distinción muy marcada entre lo teórico y lo práctico, de manera que pudiéramos decir que en la práctica administrativa ella se propone controlar (para bien o para mal) los fenómenos administrativos.

8. Lo que puede concluirse, sin recurrir a este procedimiento del autor que paradójicamente otorga cientificidad a la administración y al mismo tiempo se la quita (o sea sin la ambición de alcanzar un término medio entre lo práctico y lo teórico), es que definitivamente la teoría administrativa puede ser ciencia y lo seguirá siendo siempre y cuando trabaje desinteresadamente por el conocimiento objetivo de los fenómenos administrativos, y que la práctica administrativa, como conocimiento práctico interesado, no es científico ni llegará a serlo en ningún momento.g: 